\title{
FACTORS AFFECTING THE OUTCOME OF CHILDREN TREATED FOR AMBLYOPIA
}

\author{
G. WOODRUFF, F. HISCOX, J. R. THOMPSON and L. K. SMITH \\ Leicester
}

\begin{abstract}
SUMMARY
The outcome of treatment for amblyopia and the factors that affect this are not well understood. A major reason for this has been the exclusion from previous large studies of a sometimes unknown number of patients because of failure to comply with treatment. This paper analyses the outcome of amblyopia treatment in a retrospective review of the orthoptic records of a cohort of 961 children treated for amblyopia at seven centres who first attended in 1983. The final visual acuity was recorded by Snellen or matching methods in 894 children $(93 \%)$. Of these, $48 \%$ achieved $6 / 9$ or better, $35 \%$ less than $6 / 9$ but better than or equal to $6 / 18$, and $17 \%$ achieved less than $6 / 18$. The outcome was best for pure anisometropic amblyopia, intermediate for pure strabismic amblyopia and least good for mixed strabismic and anisometropic amblyopia with a final visual acuity of 6/10.2, 6/12.8 and 6/14.8 respectively. While the age at start of treatment did not correlate with final visual acuity both poor initial visual acuity and poor compliance were associated with poor outcome. The main factor affecting the outcome of amblyopia treatment is the initial visual acuity. Comparison with the literature suggests that the results of treatment in this country may be falling far short of what would be possible in ideal circumstances with unlimited resources.
\end{abstract}

There is a wide range in the reported results of treatments of amblyopia with success rates ranging from $30 \%$ to $92 \%{ }^{1,2}$ Reasons for this include the selection of patients and the exclusion from some studies of those not completing treatment. ${ }^{3}$ There have been few studies with large numbers of patients which include information on children who do not complete treatment and there have been no multicentre studies. This paper analyses factors associated with the outcome of amblyopia treatment in a multicentre study of 961 children treated for amblyopia and followed for up to 10 years.

Correspondence to: Geoffrey Woodruff, FRCOphth, Department of Ophthalmology, University of Leicester, Clinical Sciences Building, Leicester Royal Infirmary, PO Box 65, Leicester LE2 7LX, UK.

\section{PATIENTS AND METHODS}

Data were collected on all 961 patients first seen at each of seven English Orthoptic Centres in 1983 who were prescribed occlusion for anisometropic, strabismic, or mixed amblyopia at any time either at the first appointment or up to 10 years subsequently. The visual acuity (including the method of measurement) and the treatment prescribed at each visit were entered into a database from the orthoptic records. The same orthoptist supervised the collection of data at each centre. Details of the presentation of these children are given in an accompanying paper. ${ }^{4}$ The degree of social deprivation of each child was estimated by the Townsend deprivation score ${ }^{5}$ using electoral ward data from the 1981 census linked to the postcode of each patient. Statistical analysis of social deprivation was based on these raw values although for tabulation the deprivation scores are grouped into quintiles.

\section{RESULTS}

\section{Outcome for Different Types of Amblyopia}

The final visual acuity, i.e. the best visual acuity within three visits of the cessation of treatment, was recorded by either matching (49\%) or Snellen methods (51\%) in 894 $(93 \%)$ of the children. Of these, $48 \%$ achieved $6 / 9$ or better, $35 \% 6 / 18$ or $6 / 12$ and $17 \%$ achieved less than $6 / 18$. The outcome was better for pure anisometropes, intermediate for pure strabismic patients and least good for mixed strabismic and anisometropic patients (Fig. 1). with a mean final visual acuity (VA) $\exp \left[\left(\sum \log \mathrm{VA}\right) / n\right]$ of $6 / 10.2(n=163), 6 / 12.8(n=477)$ and 6/14.8 $(n=254)$, respectively. This was statistically significant $(p<0.0001)$.

There was a significant relationship between difference in spherical equivalent between the two eyes and final visual acuity amongst those children with anisometropia (linear regression: $n=157, p<0.0001$ for pure anisometropes; $n=248, p<0.0001$ for mixed amblyopes), with worse final visual acuity associated with higher degrees of anisometropia.

\section{Age at Start of Treatment}

Neither the age at presentation nor the age at the start of 


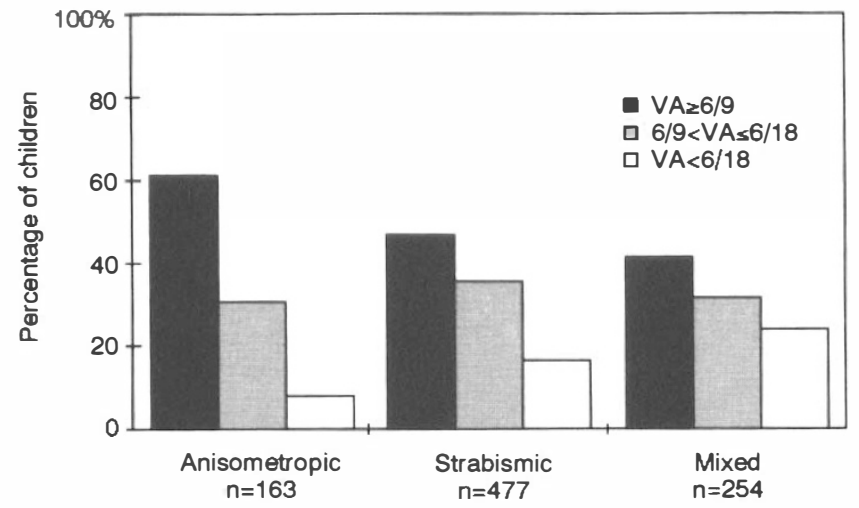

Fig. 1. Percentage of children with different levels of final visual acuity for each type of amblyopia.

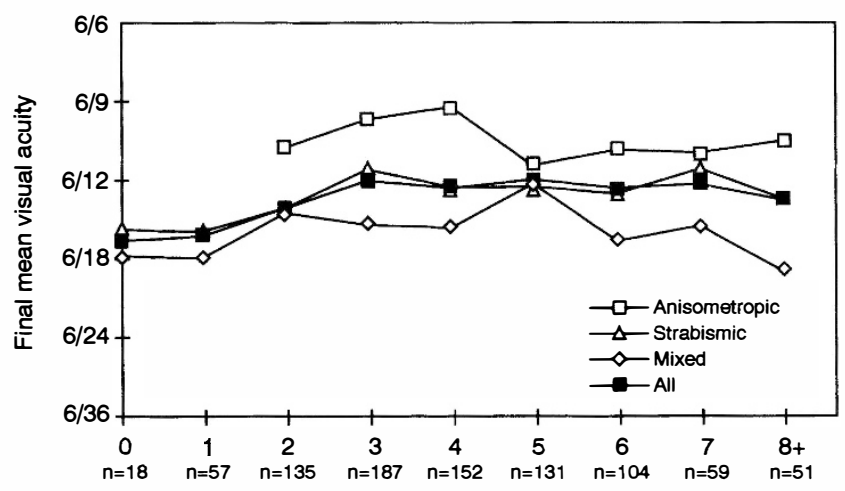

Age in years at start of treatment

Fig. 2. Mean final visual acuity and age at starting treatment for each type of amblyopia and for all types combined. There was no significant association between age at start of treatment and final visual acuity.

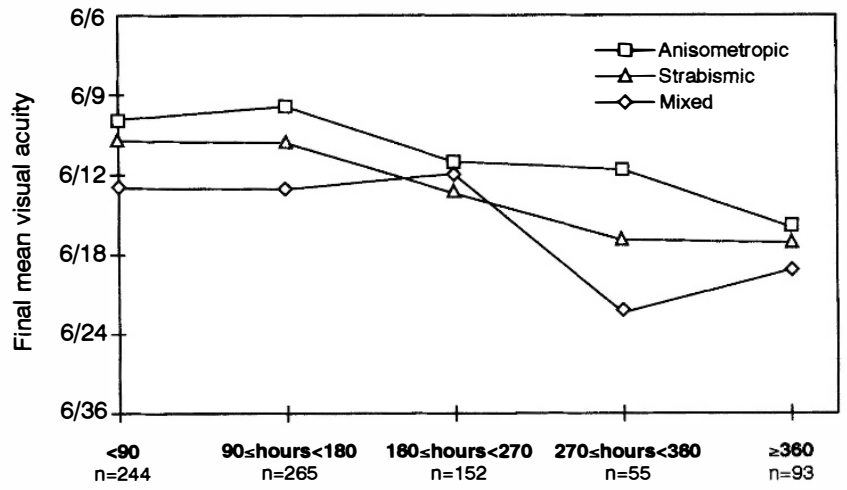

Hours of patching in the first 3 months of treatment

Fig. 4. Mean final visual acuity and hours of patching in the first 3 months of treatment.

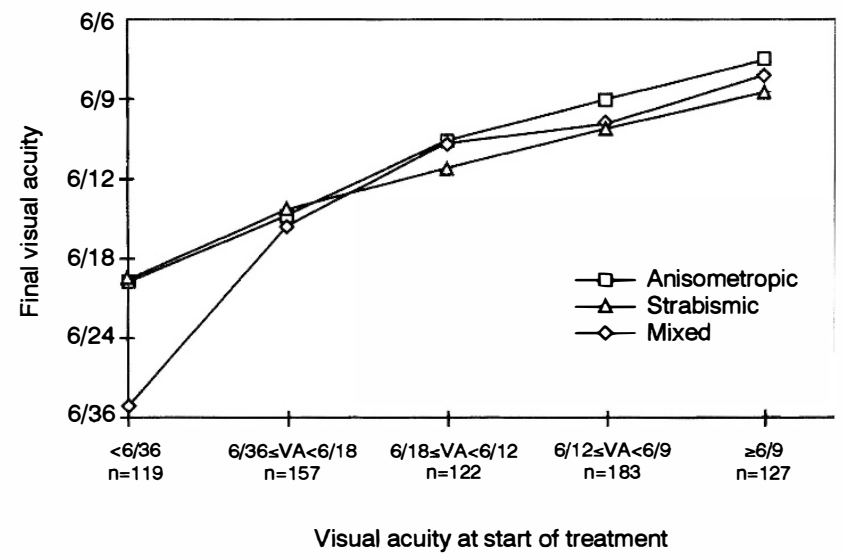

Fig. 3. Mean final visual acuity and visual acuity at start of treatment. The final visual acuity was significantly associated with the visual acuity at the start of the treatment.

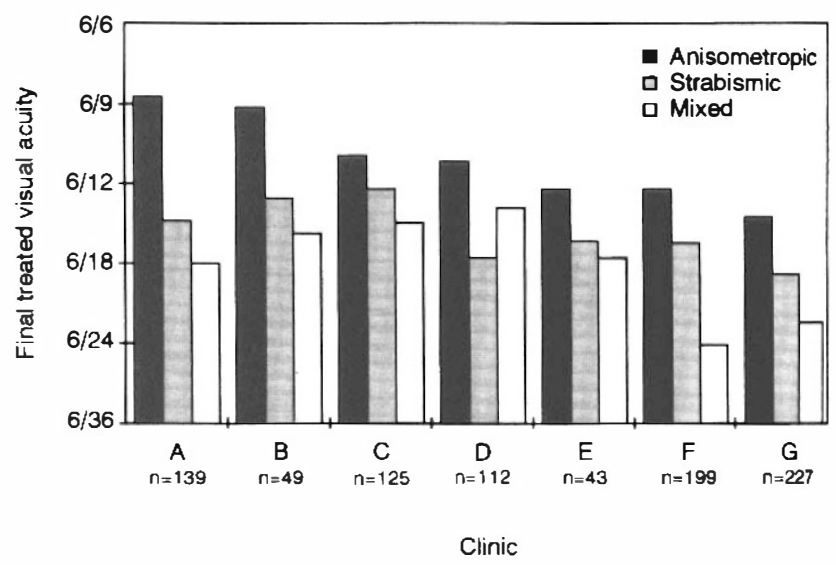

Fig. 5. Outcome of amblyopia treatment at seven centres $(A-G)$. Regression analysis showed that even after allowing for differences in initial acuity there were significant differences between centres. 
treatment (Fig. 2) was associated with the final outcome. This was true whether children with all types of amblyopia were considered together (age at start of treatment, $p=0.08$ ) or analysed separately (anisometropic amblyopia: $p=0.48$; strabismic amblyopia: $p=0.10$; mixed amblyopia: $p=0.64$ ).

\section{Final Visual Acuity and Initial Visual Acuity}

A total of 708 children had a Snellen or matching acuity measurement prior to starting treatment. For each type of amblyopia the visual acuity at referral and at start of treatment correlated closely with the final visual acuity. This was statistically significant for all types of amblyopia $(n=708, p<0.0001)$ and each type of amblyopia separately (anisometropic amblyopia: $n=162, p<0.0001$; strabismic amblyopia: $n=338, \quad p<0.0001$; mixed amblyopia: $n=208, p<0.0001$ ). For children whose acuity at start of treatment was less than 6/9 the average improvement in visual acuity was 1.8 Snellen lines (Fig. $3)$.

\section{Association Between Appointments Kept and Final Visual Acuity}

There was a significant association between percentage of prescribed appointments kept during the first year of treatment and the final visual acuity $(n=894, p<0.0001)$. Patients who missed no appointments in the first year of treatment had a mean final visual acuity of 6/9.5 $(n=94)$ for anisometropic, 6/11.3 $(n=225)$ for strabismic and $6 / 14.0(n=139)$ for mixed amblyopia. The mean acuity for patients who had missed an appointment in the first year of treatment was 6/11.1 $(n=69), 6 / 14.3(n=252)$ and $6 / 15.9(n=115)$, respectively.

\section{Association Between Social Deprivation and Outcome}

There was no significant relationship between social deprivation measured using the Townsend score and final visual acuity amongst children with pure anisometropic and mixed amblyopia $(n=163, p=0.45 ; n=253$, $p=0.33$, respectively). However, there was a slight association between social deprivation and outcome for patients with strabismic amblyopia $(n=477, p=0.04)$ with the most deprived quintile of strabismic children having a mean final visual acuity of 6/14.4 compared with $6 / 11.7$ for the least deprived quintile.

\section{Hours of Patching and Final Visual Acuity}

In $80 \%$ of the children the number of hours of patching prescribed in the first 3 months of treatment was recorded. There was a highly significant relationship between hours of patching prescribed in the first 3 months and final visual acuity for all types of amblyopia $(n=809, p=0.0001)$ and for each type of amblyopia analysed separately (Fig. 4) (anisometropic: $n=152, \quad p=0.008$; strabismic: $n=423, p=0.007$; mixed: $n=234, p=0.004)$. On average children who were prescribed less than 90 hours of patching in the first 3 months of treatment had a final visual acuity of $6 / 10.8(n=244)$, while children who were prescribed 360 or more hours of patching had a mean final visual acuity of 6/16.7 ( $n=93)$. However, this association could be attributed to the greater number of hours of patching prescribed for children with poorer initial visual acuities (regression analysis of final visual acuity adjusted for initial visual acuity: anisometropic: $n=152, p=0.31$; strabismic: $n=299, p=0.81$; mixed: $n=193, p=0.90$ ).

\section{Factors Associated with Clinic}

Even after adjusting for all other variables there remained significant differences in outcome at the different centres $(p=0.02)$ (Fig. 5). We therefore analysed the characteristics of different centres to see whether these related to outcome. The following factors were considered: average age of presentation of each type of amblyopia to each centre; mean number of visits in the first year for each type of amblyopia; and proportion of patients who missed appointments at that clinic in the first year.

It was found that children with anisometropia from centres with a younger mean age of starting treatment had a significantly better final visual acuity than those from centres with an older mean age $(p=0.001)$. There was a similar but marginally non-significant effect amongst those with mixed amblyopia $(p=0.06)$. Children with strabismus showed no similar relationship $(p=0.33$ ).

There was no evidence of a relationship between the number of visits prescribed by each centre and final visual acuity amongst those children with strabismic or mixed amblyopia ( $p=0.68$ in each case). However, for children with anisometropic amblyopia centres which prescribed, on average, more visits to their patients had better results $(p=0.003)$.

\section{DISCUSSION}

The outcome of amblyopia treatment is notoriously difficult to evaluate. ${ }^{6}$ There are problems with the mathematical analysis of acuity data, the small size of most of studies, and with the selection of patients.

In analysing data of children with amblyopia there are two problems not encountered in analysing acuity data from adults. Firstly, the visual acuity of children, when tested by the same method, tends to improve with age; secondly, young children are usually tested with the Sheridan Gardiner chart while older children are tested with the Snellen chart and these two tests are not equivalent. Several methods ${ }^{7,8}$ have been suggested to overcome these problems, the most satisfactory being the acuity ratio of Fulton et al. ${ }^{9}$ which is calculated by reference to the acuity of the non-amblyopic eye. In our series a visual acuity of better than $6 / 6$ was almost never recorded and thus we were not confident that the minimum angle of resolution of the non-amblyopic eye had been accurately recorded. Better analysis of our data would have been possible if more accurate testing of the acuity in the better eye had been done.

In the largest series of amblyopic patients to date, 
Massie ${ }^{10}$ reported on 949 patients treated at one centre. However, the method of selection of patients, particularly with regard to patients who failed to attend, is not clear. Since more than two thirds of the patients were aged 7 years or more when treatment started, it is unlikely that his series is representative of patients treated at most clinics in North America and Britain today. Similarly, Bremner ${ }^{11}$ reported on 240 patients treated with the Cam stimulator, but the results of only $42 \%$ of the patients initially started on treatment are recorded in her study and nearly half of these were more than 8 years of age. More satisfactory studies of amblyopia outcome such as those by Kutschke et al. ${ }^{12}$ Fulton and Mayer, ${ }^{9}$ Neumann et al. ${ }^{8}$ and Litthander and Sjöstrand ${ }^{13}$ have reported on smaller numbers of patients and, with the exception of Litthander and Sjöstrand,$^{13}$ these authors have all specifically excluded patients who failed to comply with treatment.

Overall $48 \%$ of the patients in this study achieved $6 / 9$ or better visual acuity. This compares poorly with the more than $80 \%$ of the patients of Fulton \& Mayer and of Ching et al. ${ }^{14}$ who achieved $6 / 9$ or better and the $83 \%$ of the patients of Kutschke et al. ${ }^{12}$ with anisometropic amblyopia who achieved 20/40 or better. At least part of this difference can be attributed to the exclusion of non-compliant patients in these other series. The results of treatment of our fully compliant patients were better, with $52 \%$ of the fully compliant patients achieving 6/9, compared with $44 \%$ of those who missed appointments. However, Lithander and Sjörstrand ${ }^{13}$ have reported the results of treatment of 44 consecutive children treated for amblyopia in the context of private practice and weekly follow-up. In this series no patients were excluded because of failure to attend. Lithander and Sjörstrand's ${ }^{13}$ results suggest that with ideal treatment and generous resources a success rate of nearly $100 \%$ can be aimed for rather than the $50 \%$ recorded from the centres we have studied in the United Kingdom.

Despite the large numbers in our series we, like previous authors, could find no significant association between young age at presentation and better outcome. In fact there was a tendency for the reverse to be true. The time of onset of strabismus, and therefore of strabismic amblyopia at least, varies widely between children. We found a marked correlation between initial visual acuity and outcome, showing that poor initial visual acuity indicates severe (i.e. difficult to treat) amblyopia. We suspect that later onset amblyopia tends to be less severe than earlier onset amblyopia, and also that during any delay between onset and start of treatment there is a progressive deterioration towards more severe amblyopia. These two factors could result in the finding of no overall correlation between age and outcome.

Like Lithander and Sjörstrand ${ }^{13}$ we found that good compliance was associated with better outcome. Since social deprivation did not make much difference to outcome, we suspect that their system of intensive follow-up contributed to better compliance in their series and was the main factor responsible for the difference in overall out- come in the two series. Patients prescribed more hours of patching in the first year of treatment had a worse outcome than children prescribed fewer hours. For all children we demonstrated that this association could be attributed to the fact that more hours of patching were prescribed for children with worse initial visual acuities.

Overall we conclude that the main factor affecting the outcome of amblyopia treatment is the initial visual acuity. If differences in the severity of amblyopia at the start of treatment are taken into account the mean outcome for children with strabismic amblyopia at the different centres in our study was similar. However, there remained differences in outcome of anisometropic and mixed amblyopia and those centres having less good results with these types of amblyopia were those where patients with anisometropia presented late. It is not clear whether this is because the centres with the better results treated patients sooner after the onset of amblyopia than the centres with the less good results or whether the treatment itself was indeed better at these centres. For better analysis of treatment in future studies the visual acuity of the good eye should be recorded accurately. However, it appears that the outcome of amblyopia treatment in many centres in this country is worse than it could be in ideal circumstances with unlimited resources.

We thank Jackie Nolan, Liz Newcombe, Rowena McNamara, Susan Viney, Jenny Elmer-Monaghan, Sheelagh Baker and the staff of the Orthoptic Clinics at Birmingham Children's Hospital, Bristol Eye Hospital, Leeds General Infirmary, Leicester Royal Infirmary, Queen's Medical Centre Nottingham, Sunderland Eye Infirmary and Worthing Hospital. We gratefully acknowledge the financial support of the British Council for the Prevention of Blindness, The Iris Fund for Prevention of Blindness, The British Orthoptic Society and the Anne Allerton Fund.

\section{REFERENCES}

1. Flynn JT, Cassady JC. Current trends in amblyopia therapy. Ophthalmology 1978;85:428-50.

2. Scott WE, Dickey CF. Stability of visual acuity in amblyopic patients after visual maturity. Graefes Arch Clin Exp Ophthalmol 1988;226:154-7.

3. Hiscox FA, Strong N, Thompson JR, Minshull C, Woodruff G. Occlusion for amblyopia: a comprehensive survey of outcome. Eye 1992;6:300-4.

4. Woodruff G, Hiscox F, Thompson JR, Smith LK. The presentation of children with amblyopia. Eye 1994;8:623-6.

5. Townsend P, Phillmore P, Beattie A. Health and deprivation: inequality and the North. London: Croom Helm, 1988.

6. Romano PE. Needed: better outcome evaluation of treatment of amblyopia and strabismus. [editorial]. Binocular Vis Q 1991;6(2):71-2.

7. Meyer E, Mizrahi E, Perlman I. Amblyopia Success Index:a new method of quantitative assessment of treatment efficiency; application in a study of 473 anisometropic amblyopic patients. Binocular Vis Q 1991;6(2):83-90.

8. Neumann R, Oliver M, Gottesmann N, Shimshoni M. Prognosis for occlusive therapy for strabismic and anisometropic amblyopia and for different initial depths of amblyopia. Chibret Int J Ophthalmol 1989;6(3):22-6.

9. Fulton AB, Mayer DL. Esotropic children with amblyopia. effects of patching on acuity. Graefes Arch Clin Exp Ophthalmol 1988;226:309-12.

10. Massie H. Fixing eye occlusion: survey of approximately 1000 case histories of patients who received occlusion of the fixing eye. Trans Ophthalmol Soc Aust 1965;24:39-46.

11. Bremmer MH. Visual acuity in the primary school child 
aged four to twelve years: a review of amblyopia treatment in this age group at Princess Margaret Hospital. Aust J Ophthalmol 1984;12:395-9.

12. Kutschke PJ, Scott WE, Keech RV. Anisometropic amblyopia. Ophthalmology 1991;98:258-63.

13. Lithander J, Sjörstrand J. Anisometropic and strabismic amblyopia in the age group 2 years and above: a prospective study of the results of treatment. Br J Ophthalmol 1991;75: 111-6.

14. Ching FC, Parks MM, Friendly DS. Practical management of amblyopia. J Pediatr Ophthalmol Strabismus 1986;23: $12-6$. 